\title{
Correction to: Northerly wind trends along the Portuguese marine coast since 1950
}

\author{
Francisco Leitão ${ }^{1}$ (1) $\cdot$ Paulo Relvas $^{1} \cdot$ Fernando Cánovas $^{1} \cdot$ Vânia Baptista $^{1} \cdot$ Alexandra Teodósio $^{1}$
}

Published online: 24 April 2019

(C) Springer-Verlag GmbH Austria, part of Springer Nature 2019

\section{Correction to: Theoretical and Applied Climatology https://doi.org/10.1007/s00704-018-2466-9}

The original version of this article unfortunately contained mistakes. All figure captions are not accurate. Also, there is a repeated error in most figures because the vertical scales incorrectly show "Wind Strenght" instead of "Wind Strength" and in Fig. 4 (mid-panel) "2003" instead of "2004". The corrected figures are given below.

Publisher's note Springer Nature remains neutral with regard to jurisdictional claims in published maps and institutional affiliations.

The online version of the original article can be found at https://oi.org/ 10.1007/s00704-018-2466-9

Francisco Leitão

fleitao@ualg.pt

1 Ceentro de Ciências do Mar, Universidade do Algarve, Campus de Gambelas, 8005-139 Faro, Portugal 
Fig. 1 Map of the Portuguese coast, showing the areas studied and information on the satellite (left panel) and ICOADS (right panel) observation points used to extract the mean wind velocity between the surface and $200 \mathrm{~m}$ height for each area. The delimitation of each area (Northwestern - NW,

Southwestern - SW and Southern

- S) was adopted according to oceanographic studies and fisheries stocks division (ICES subdivision)

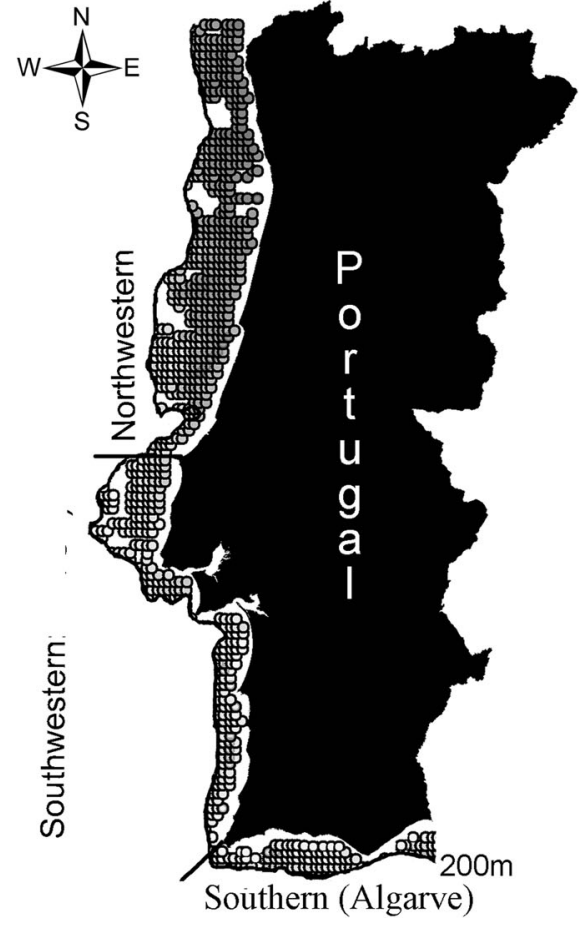

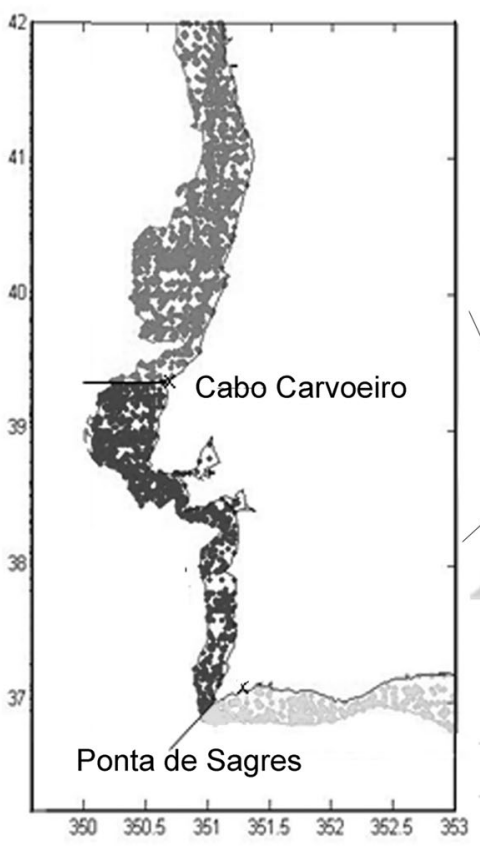


Fig. 2 a Observed and smoothed values (after LOESS) of monthly northerly wind time series intensity (v-wind) for both ICOADS and Satellite data by study area: Northwestern (NW), Southwestern (SW) and Southern (S). The horizontal line represents the average monthly v-wind values between 1960 and 2010 (ICOADS data). b Observed mean monthly v-wind values for ICOADS data between 1960 and 2010 a

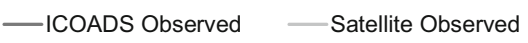

—ICOADS Smoothed — Satellite Smoothed

\section{Northwestern}

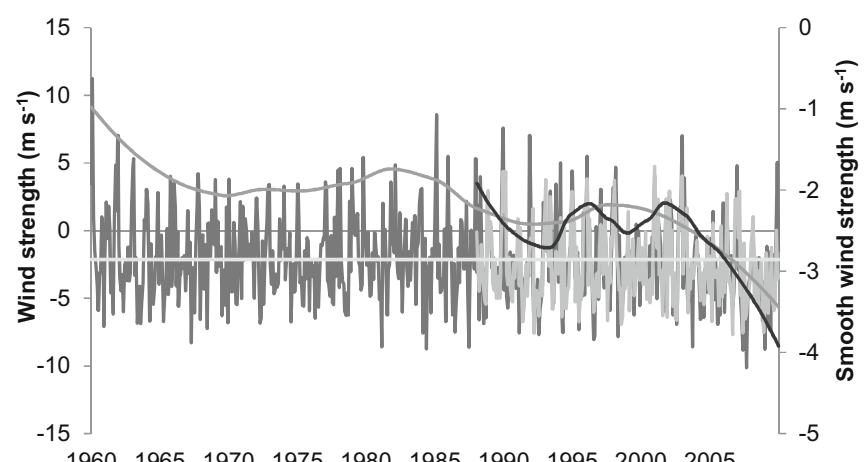

Southwestern

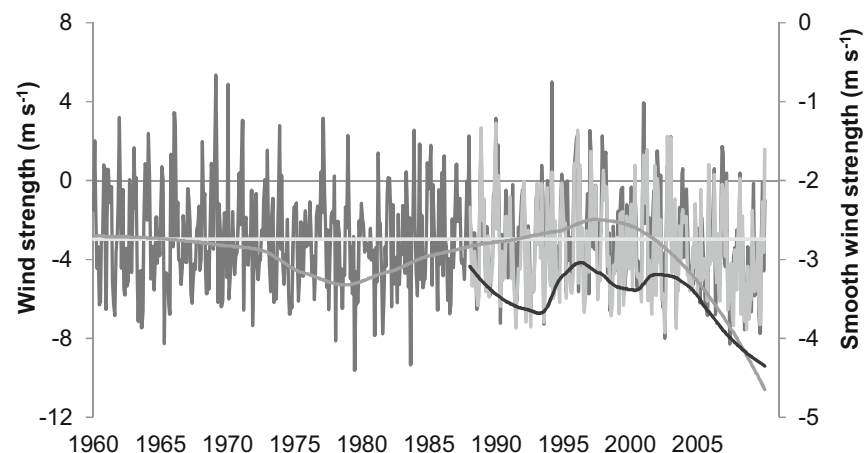

South

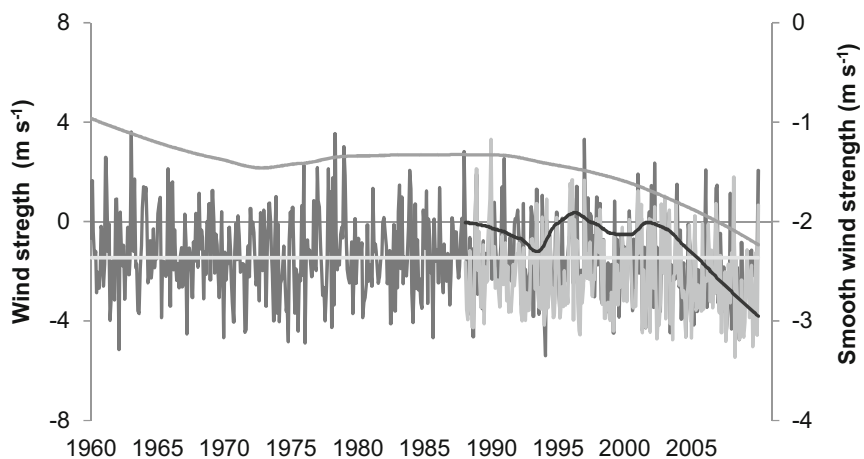

b

- -IXaCN —IXaCS -IXaS-Algarve

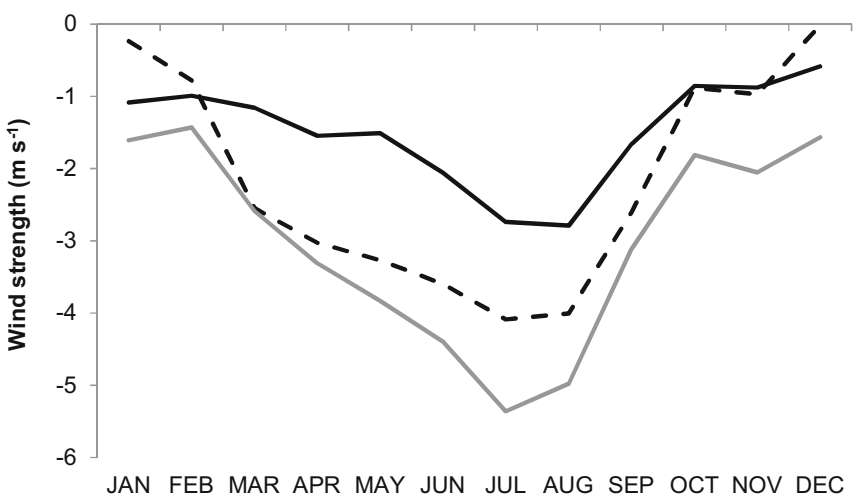

Month 

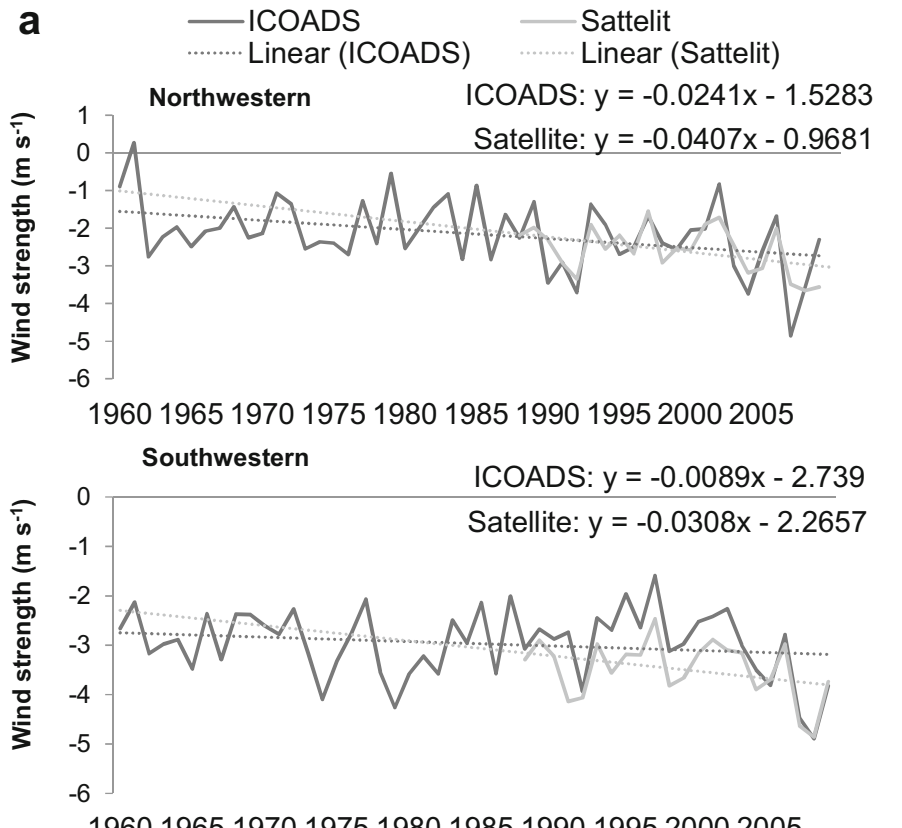

1960196519701975198019851990199520002005

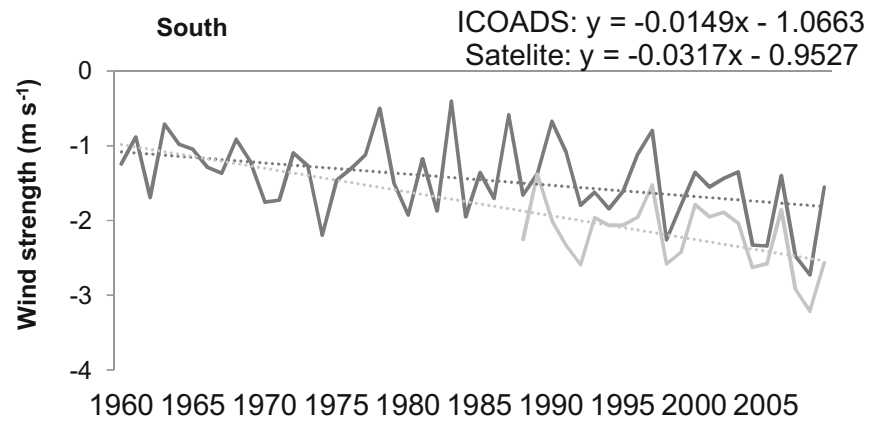

b
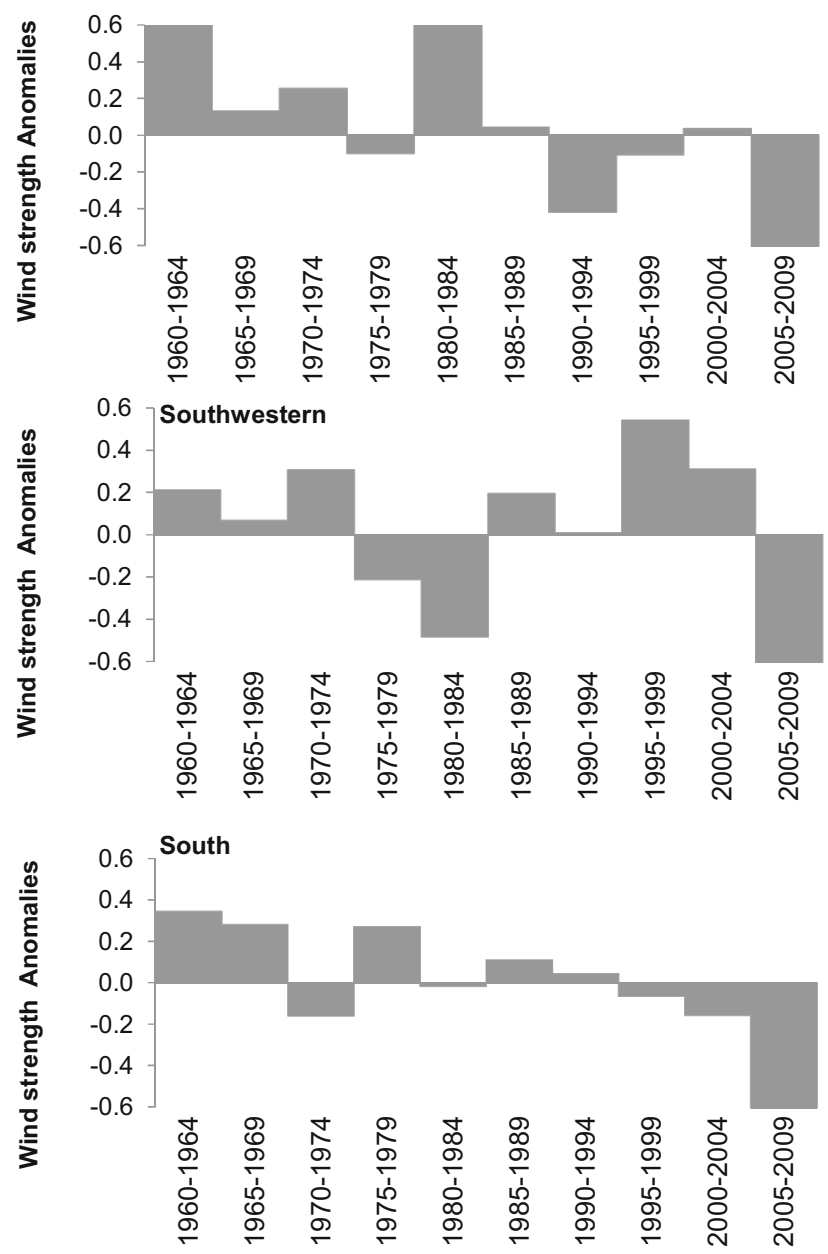

Fig. 3 a Observed and linear adjusted annual ICOADS and Satellite northerly wind (v-wind) trends by study area (left panel): Northwestern (NW), Southwestern (SW) and Southern (S). b Half-decadal anomalies in the northerly wind (v-wind) 


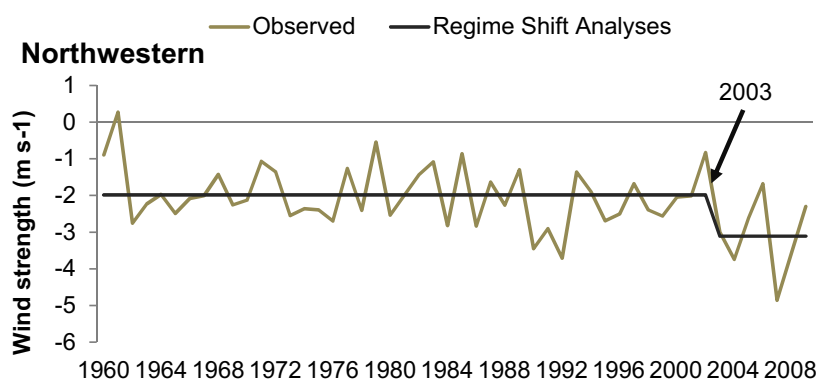

\section{Southwestern}
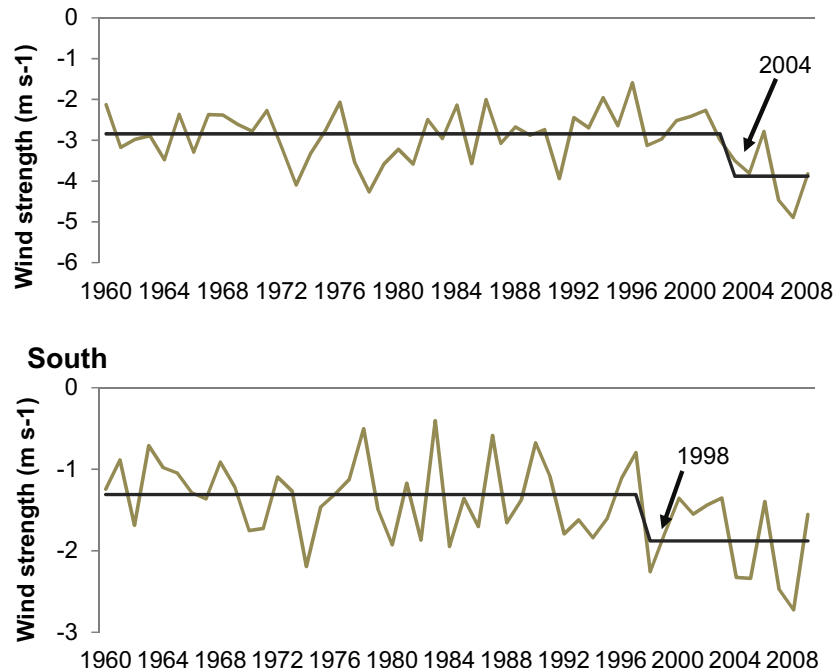

Fig. 4 Sudden shifts in the annual northerly wind (v-wind) by study area (black lines representing the RSI - Regime Shift Index): Northwestern (NW), Southwestern (SW) and Southern (S-Algarve). The significant regime shift index periods and inflection year are indicated
Northwestern

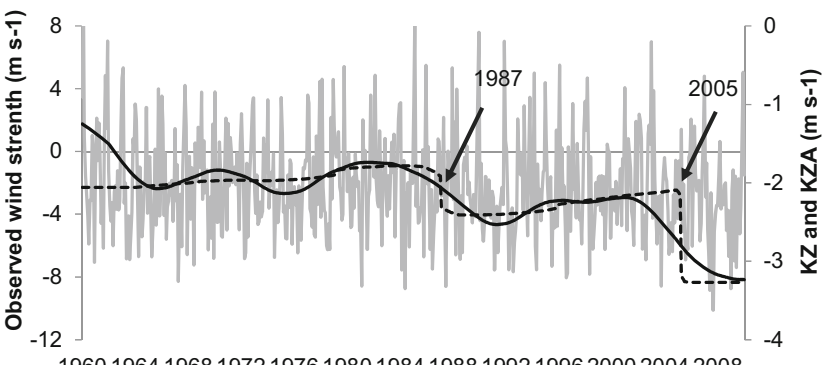

1960196419681972197619801984198819921996200020042008
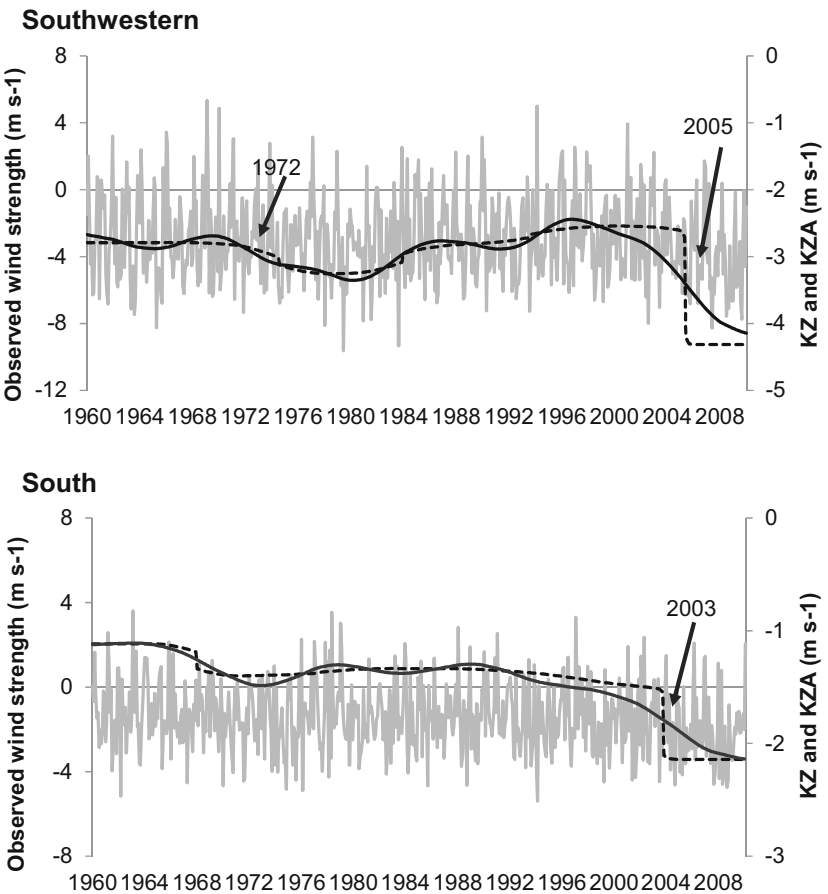

Fig. 5 Monthly observed, Kolmogrov-Zurbenko (KZ) and KolmogrovZurbenko Adaptative filter (KZA) trends in northerly wind (v-wind) sudden shifts by study area: Northwestern (NW), Southwestern (SW) and Southern (S) 
Northwestern
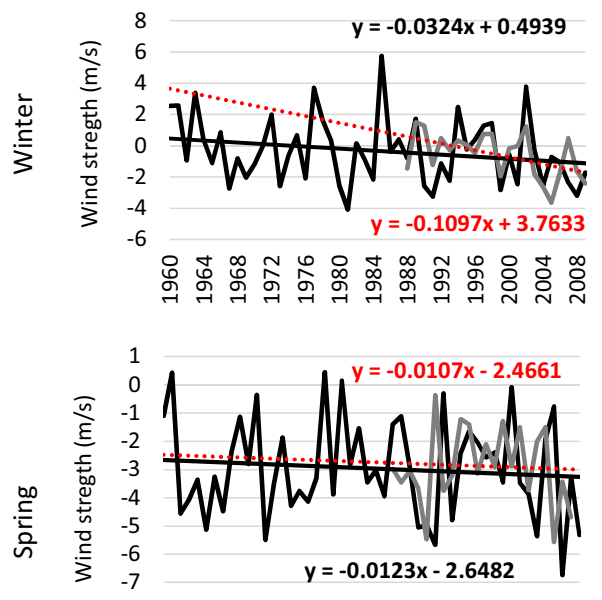

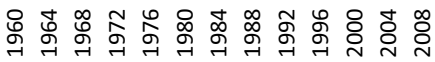

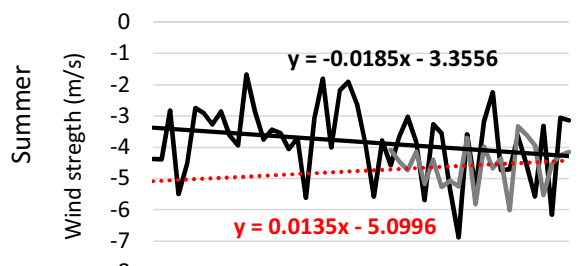

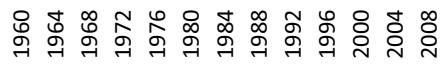

$$
\text { है }
$$

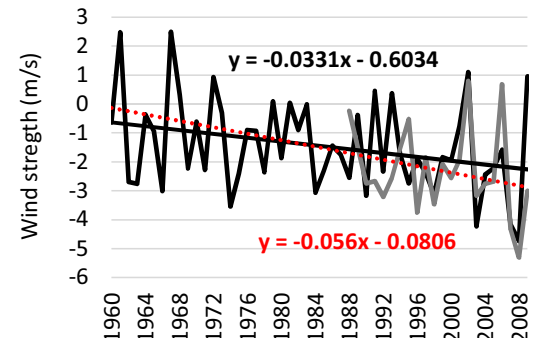

Southwestern

South
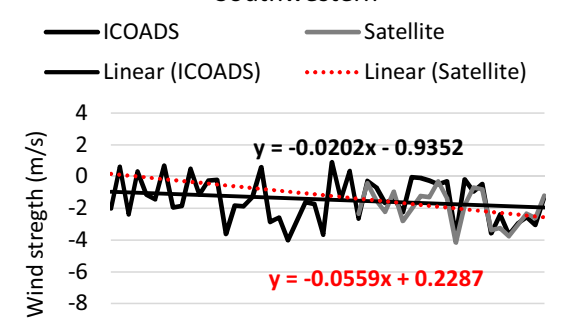

\&

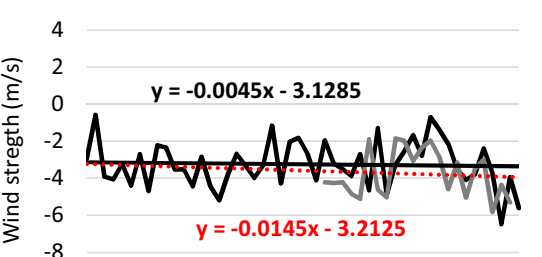

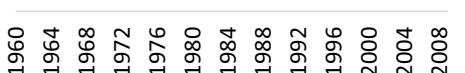

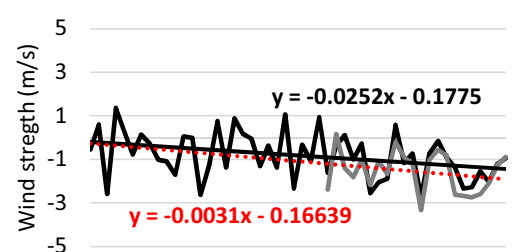

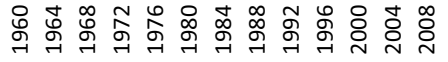
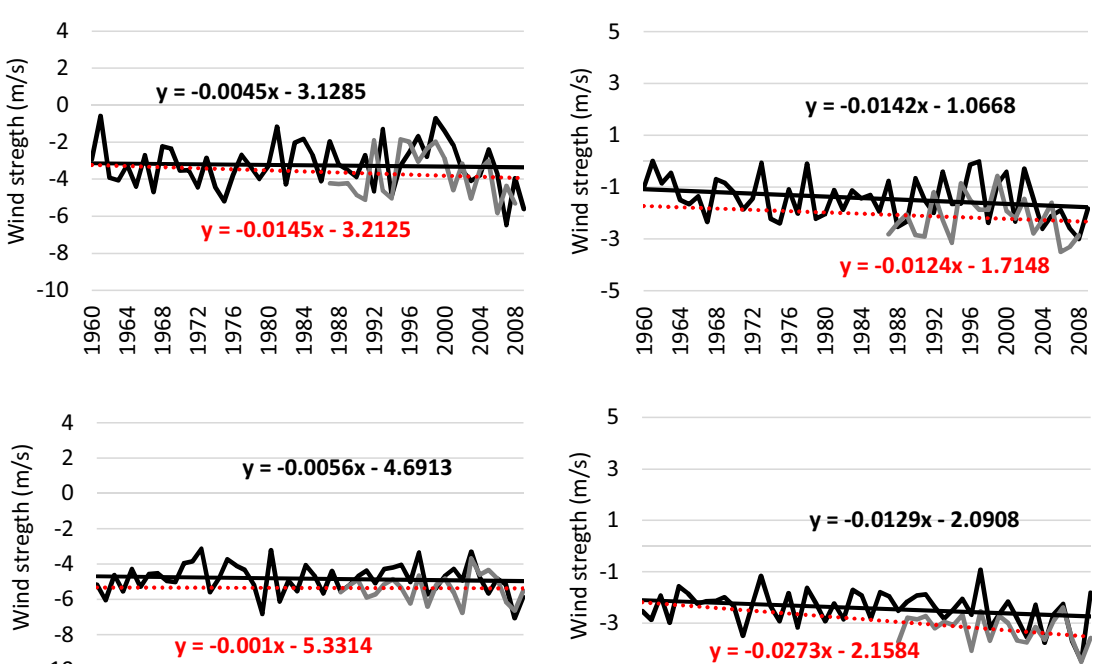

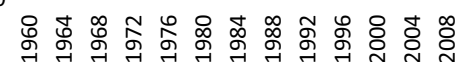
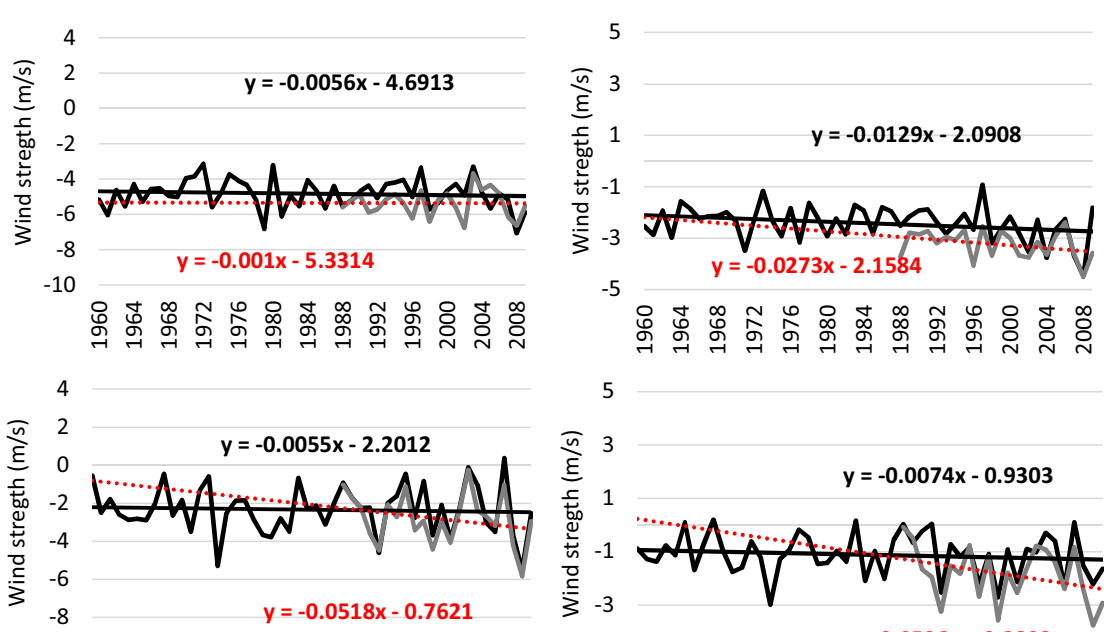

$-10$

怘志总 㣽

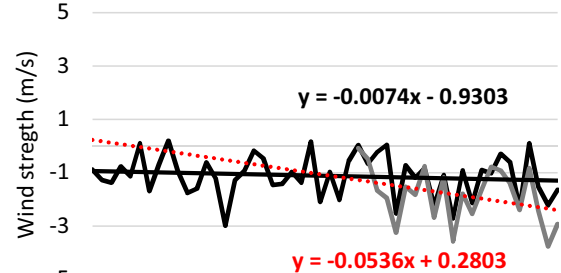

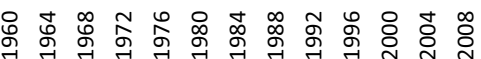

Fig. 6 Inter-annual seasonal observed and linear adjusted northerly wind (v-wind) values by study area: (a) Northwester (NW); (b) Southwestern (SW); and c) Southern (S) 


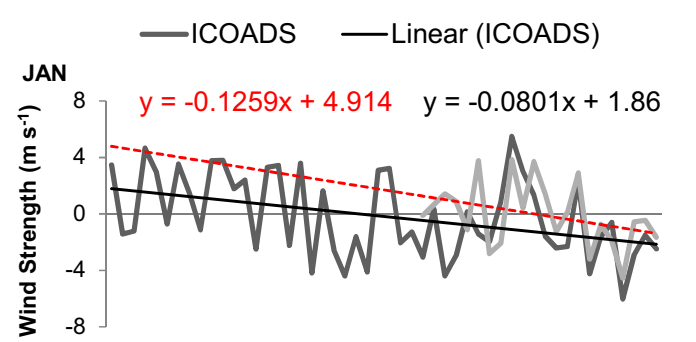

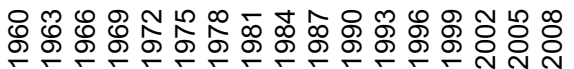

MAR $4, y=0.0792 x-5.6313 y=-0.0052 x-2.2536$

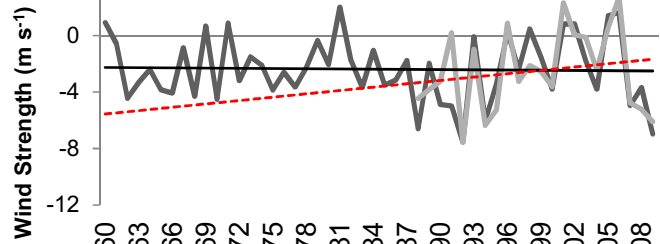

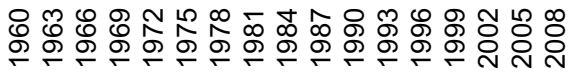

MAY $4, y=-0.1416 x+2.6248 y=-0.0113 x-2.9451$

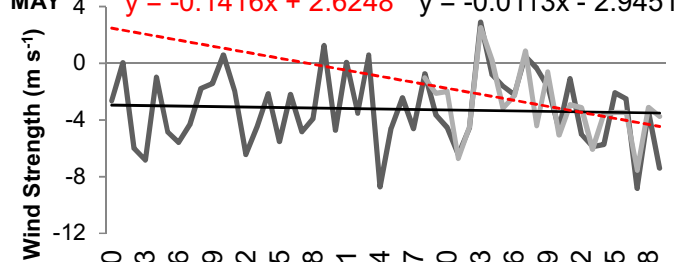

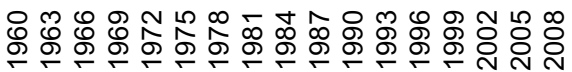

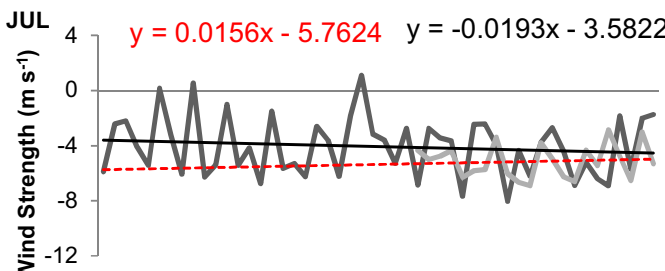

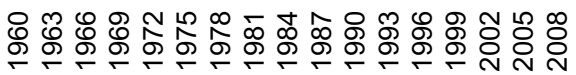

SEP 4$] y=0.0506 x-5.3607 y=-0.0586 x-1.1065$

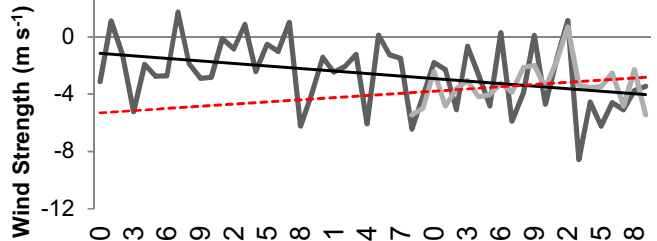

\&

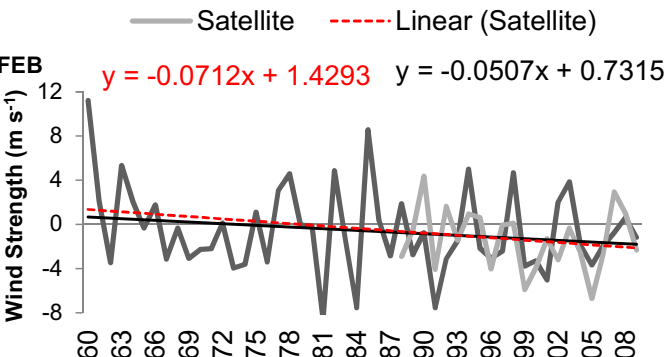

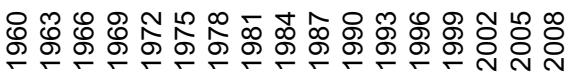

APR $8, y=-0.0203 x-2.7459 \quad y=0.0304 x-4.3599$

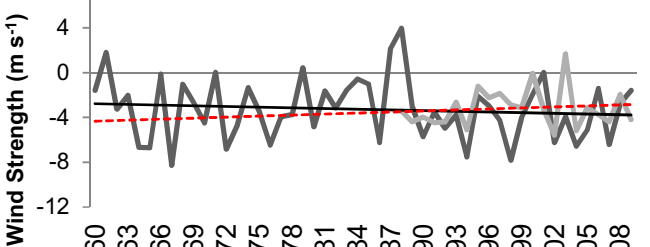

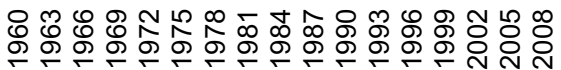

JUN 4$] y=0.0736 x-6.9551 \quad y=-0.0101 x-3.2079$

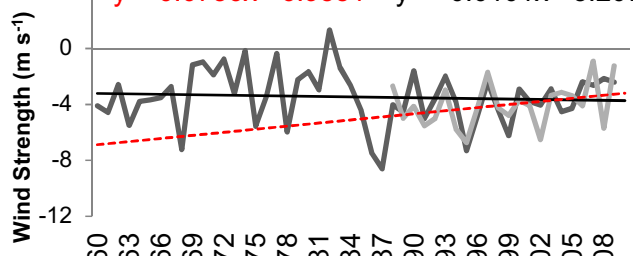

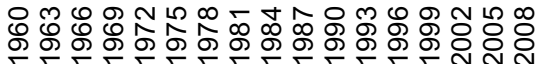

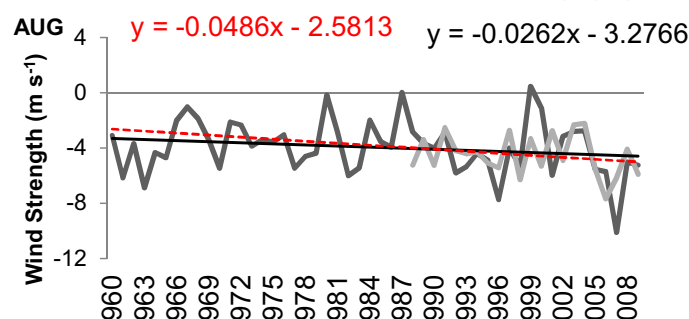

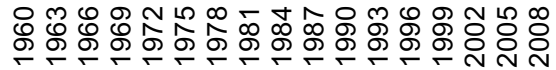

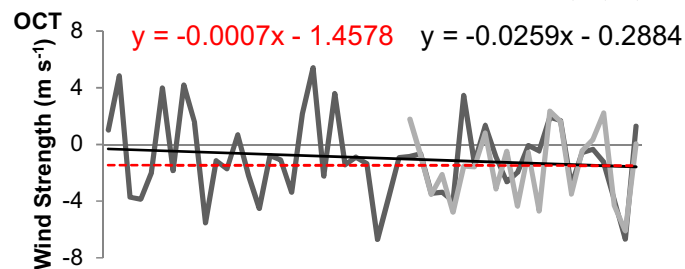

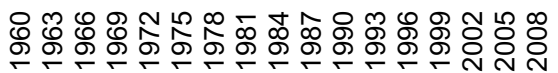
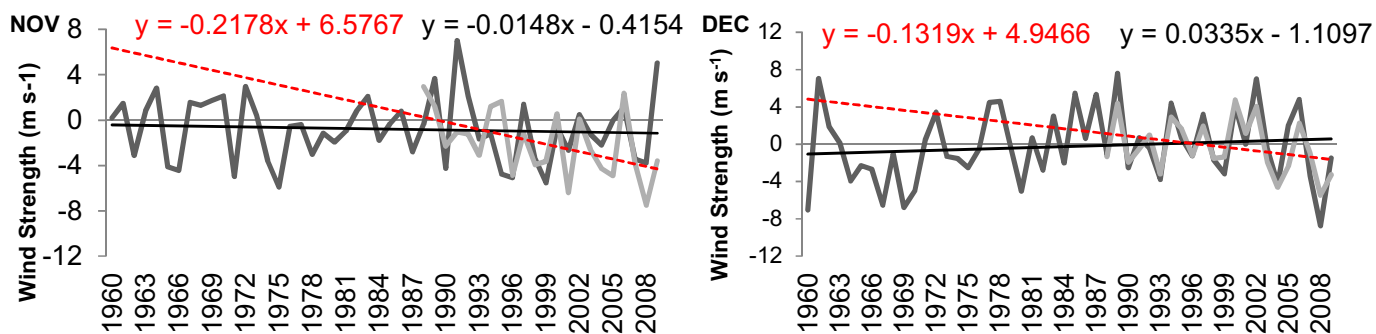

Fig. 7 Inter-annual monthly observed and linear adjusted northerly wind (v-wind) values in Northwestern (NW) coast. January; February; March; April; May; June; July; August; September; October; November; December 


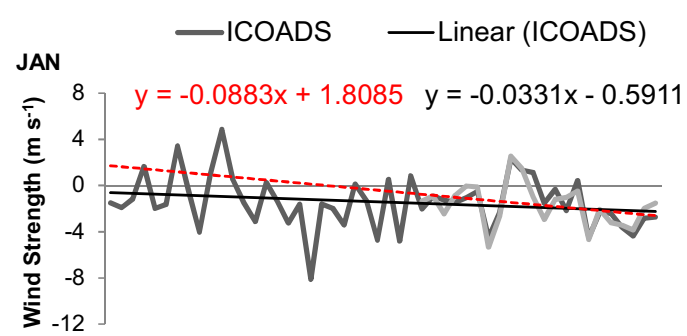

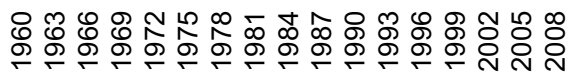

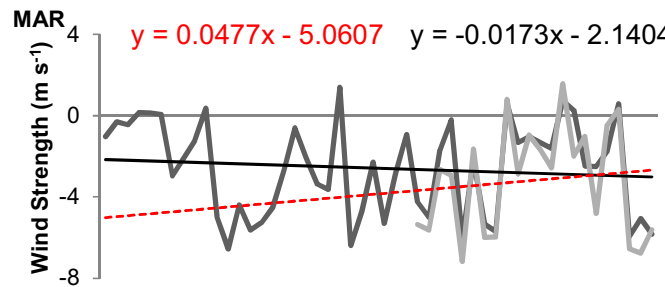

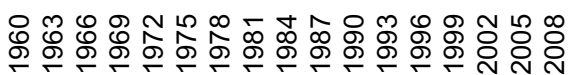

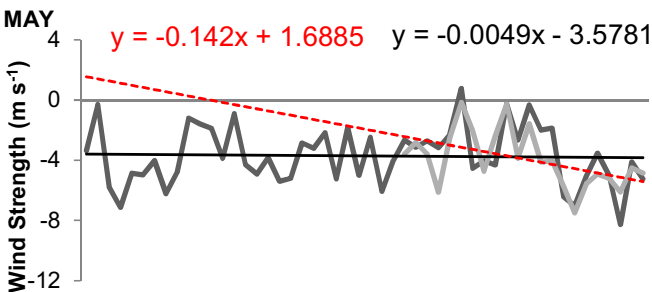

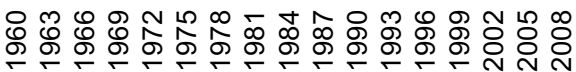

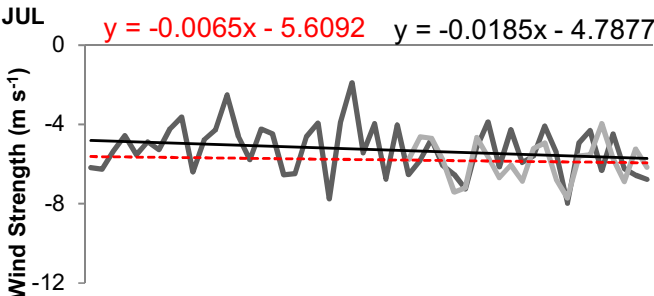

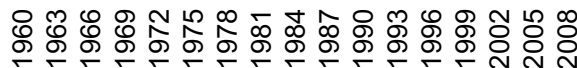

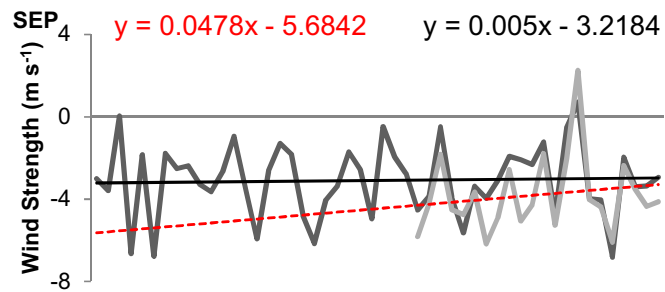

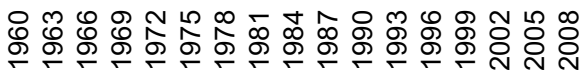

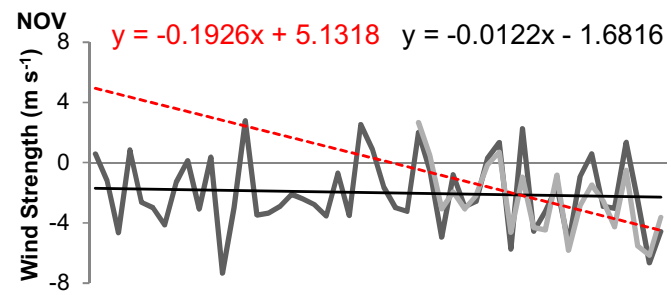

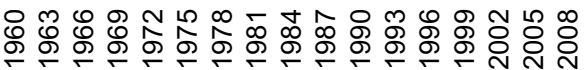

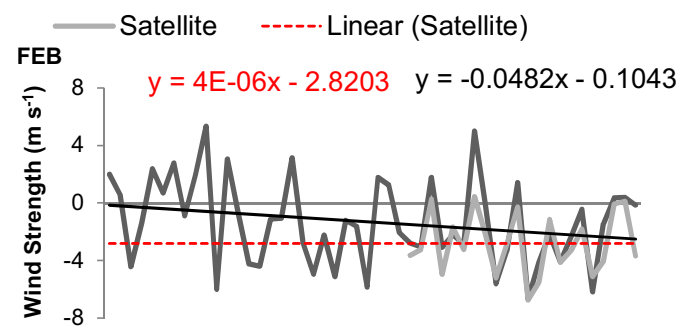

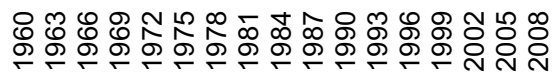

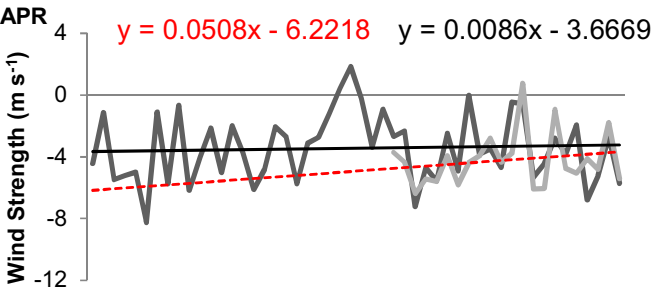

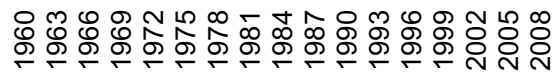

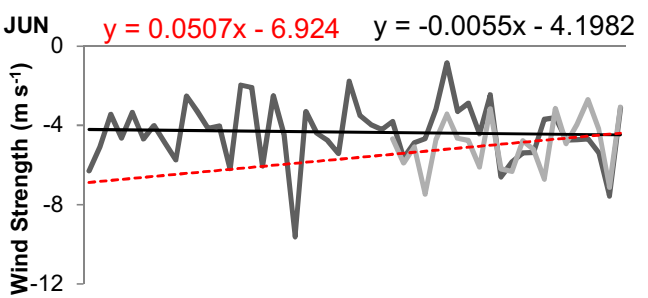

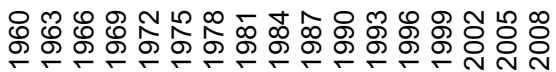

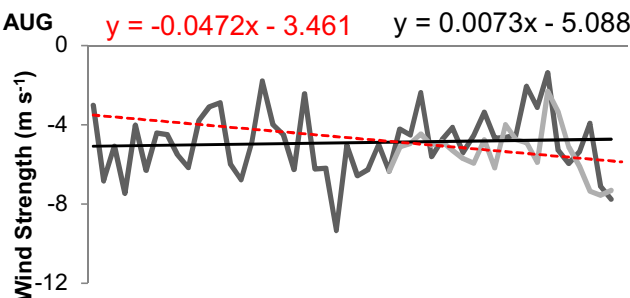

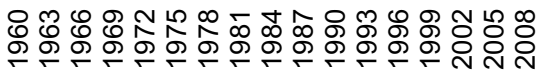

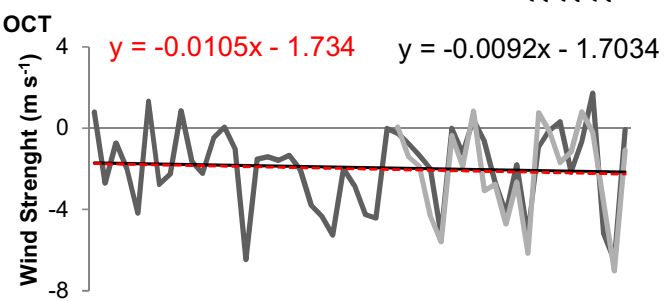

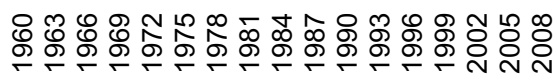

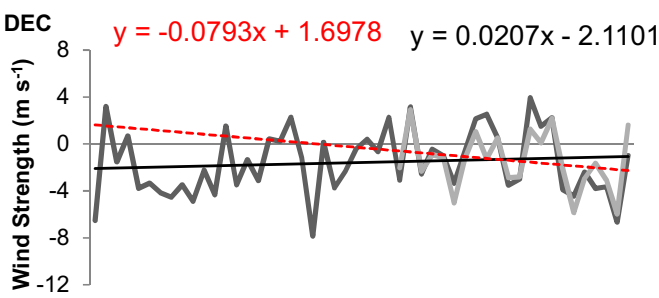

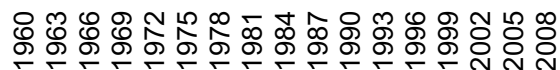

Fig. 8 Inter-annual monthly observed and linear adjusted northerly wind (v-wind) values in Southwestern (SW). January; February; March; April; May; June; July; August; September; October; November; December 

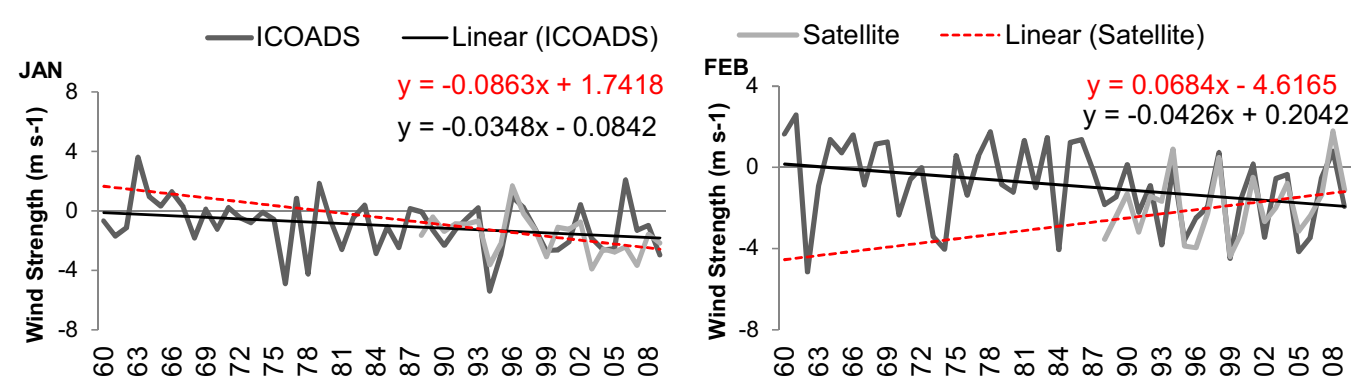

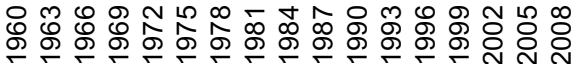

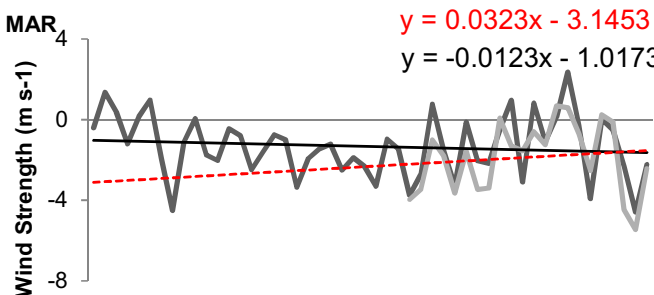

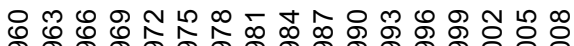

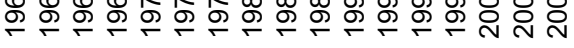

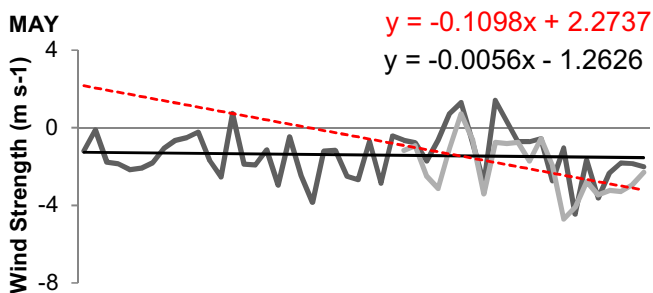

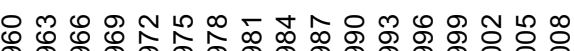

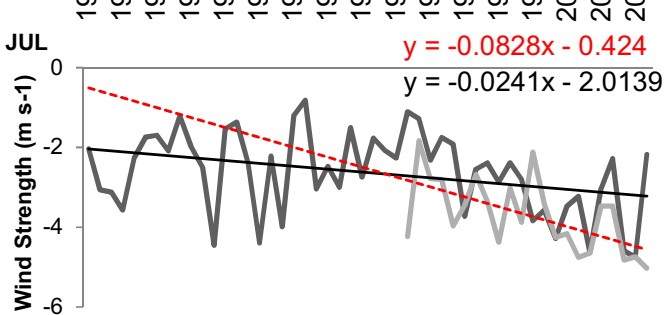

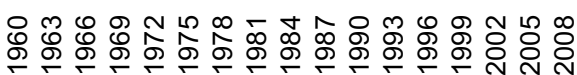

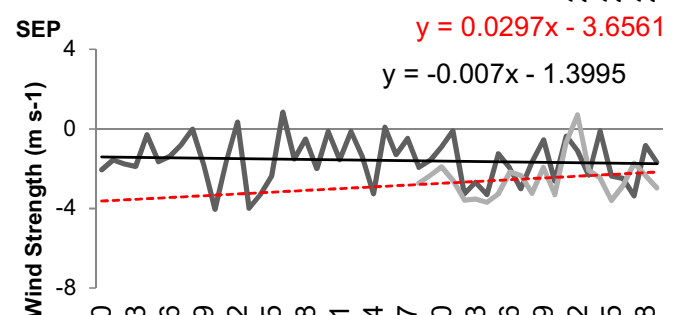

\&

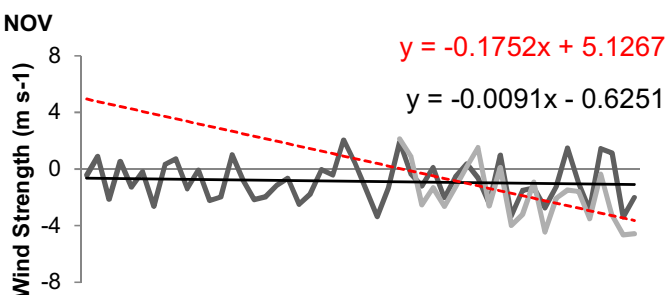

\&

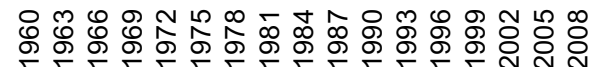

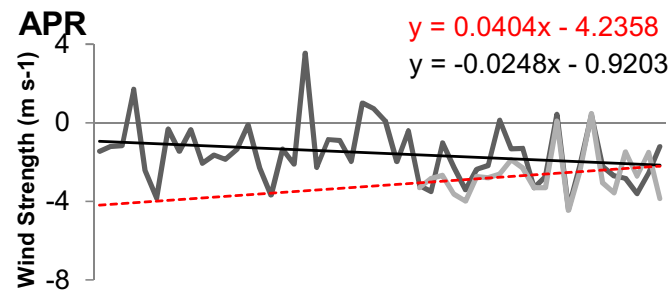

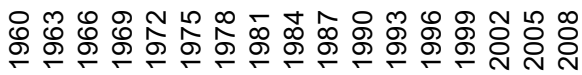

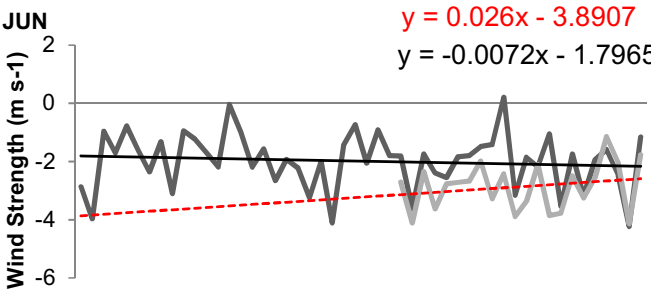

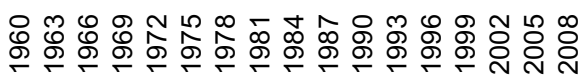
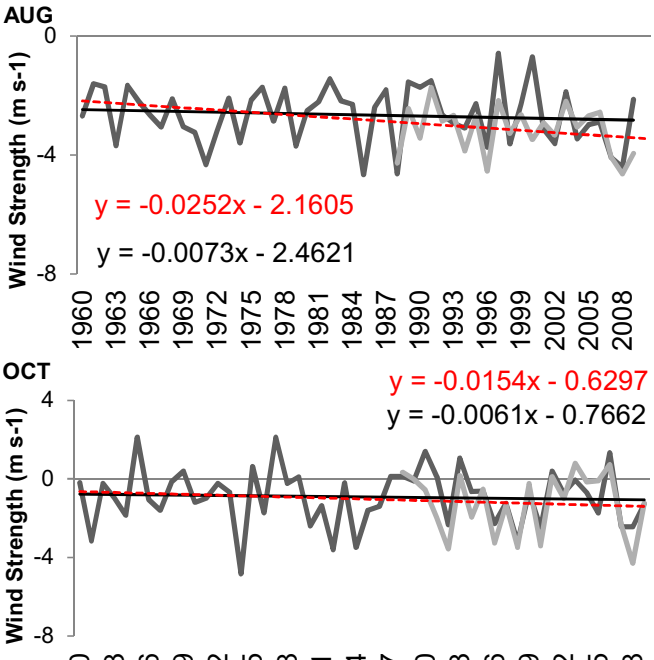

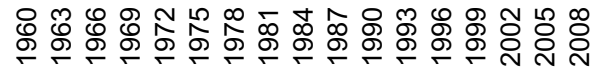
DEC

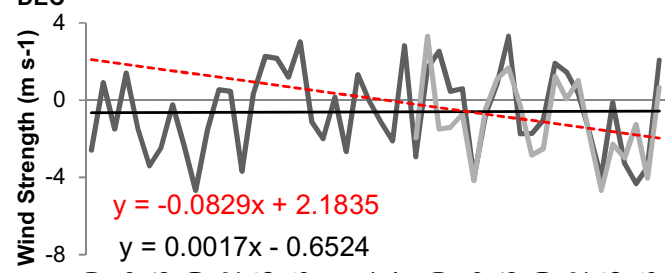

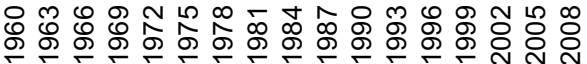

Fig. 9 Inter-annual monthly observed and linear adjusted northerly wind (v-wind) values in Southern-Algarve (S). January; February; March; April; May; June; July; August; September; October; November; December 